\title{
Learning the Relationship between High and Low Resolution Images in Kernel Space for Face Super Resolution*
}

\author{
Wilman W.W. Zou and Pong C. Yuen \\ Department of Computer Science, Hong Kong Baptist University \\ \{wwzou,pcyuen\}@comp.hkbu.edu.hk
}

\begin{abstract}
This paper proposes a new nonlinear face super resolution algorithm to address an important issue in face recognition from surveillance video namely, recognition of low resolution face image with nonlinear variations. The proposed method learns the nonlinear relationship between low resolution face image and high resolution face image in (nonlinear) kernel feature space. Moreover, the discriminative term can be easily included in the proposed framework. Experimental results on CMU-PIE and FRGC v2.0 databases show that proposed method outperforms existing methods as well as the recognition based on high resolution images.
\end{abstract}

\section{Introduction}

Face recognition from surveillance camera has wide range of applications, ranging from single standalone camera domestic application to multiple network camera law enforcement [11]. In all these applications, camera is normally installed in a way that viewing area is maximized and the face region is very small. Moreover, the person to be recognized is normally not cooperative. Therefore, to recognize a face from surveillance camera, we need to handle low resolution face image with variations, such as pose, illumination and occlusion.

Super-resolution (SR) for face image (face hallucination [1]) algorithms have been proposed to enhance the resolution of the face image for recognition [3] [4]. Theoretically, applying SR technique on the lowresolution (LR) face image, the reconstructed highresolution (HR) image can be used for face recognition. This approach works well only if the input face image is frontal and captured under good illumination.

\footnotetext{
*This work has been accepted by ICPR 2010
}

In order to normalize the face image variations to frontal view before applying the SR method, a twostep approach has been proposed. Li and Lin [7] made use of a view-based model to normalize the pose variations before the SR procedure. Jia and Gong [5] synthesized the HR images with different poses, lighting conditions and expressions based on tensor space. 3Dmodel are also adopted for handling the face variations. Mortazavian et al. [9] employed the 3DMM model to synthesis different pose images. Yu et al. [10] modeled pose and illumination using bilinear function, and employed pose-illumination-based SR method to reconstruct images. However when the face image resolution is smaller than $20 \times 20$ pixels, the results of these normalization algorithms may not be satisfactory.

To overcome the problems in face recognition from video, this paper proposes a kernel based face super resolution algorithm. With the highly complex and nonlinear face image variations, the relationship between LR and HR images will be nonlinear. It is well-known that the nonlinear kernel mapping could transform complex distributed data into high dimensional feature space where the data becomes linear separable. In this way, by conducting the relationship learning in kernel feature space, their nonlinear relationship can be learnt. Details are discussed in next section.

\section{Proposed Super Resolution with Face variations}

Figure 1 shows the block diagram of the proposed method. The proposed method consists of two phases, namely training and recognition. In training phase, given a set of LR and HR image pairs, both images are mapped to the kernel feature space by nonlinear mapping $\Phi_{L}$ and $\Phi_{H}$. The nonlinear relationship between HR and LR images can be better modeled after mapping. Then, we would like to learn their relationship $\left(R^{* *}\right)$ for super resolution from recognition perspec- 
tive. A kernel subspace based regression relationship learning is designed to estimate the relationship operator $R^{* *}$. Also, a discriminative cost function is designed and easily integrated with the learning processing, so that the relationship learning can better handle the face variations. In recognition phase, given the query image, its HR image representation in kernel space can be generated using $R^{* *}$. Then, any kernel-based face recognition methods, such as Kernel PCA [6] and Kernel Direct Discriminative Analysis (KDDA) [8] can be employed for recognition. In the following, we will first report our proposed framework. The key issues in the proposed method namely, learning the relationship $R^{* *}$ and designing the discriminative term, will be followed.

\subsection{Relationship Learning Framework for Su- per Resolution}

Let $I_{h}$ and $I_{l}$ be the high-resolution (HR) image and low-resolution (LR) image respectively. We assume that $I_{l}$ and $I_{h}$ have been aligned, so

$$
I_{l}=D I_{h}+n
$$

where $D$ is the downsampling operator, and $n$ is noise. To reconstruct the HR image from the corresponding LR one, we have proposed a novel framework to learn this relationship [13]. Suppose the relationship between HR and LR image is $\mathcal{R}$. That means for each LR image, we have

$$
\tilde{I}_{h}=\mathcal{R}\left(I_{l}\right)+n
$$

Therefore after determining the relationship $\mathcal{R}$, the HR image can be easily reconstructed by Eq.(2). To evaluate $\mathcal{R}$, the error between the reconstructed HR image and the original HR image should be minimized as follows,

$$
\mathcal{R}=\arg \min _{\mathcal{R}^{\prime}} \sum_{i=1}^{N}\left\|\mathcal{R}^{\prime}\left(I_{l}^{i}\right)-I_{h}^{i}\right\|^{2}
$$

where $\left(I_{l}^{i}, I_{h}^{i}\right)$ is the $i$-th training image pair, and $N$ is number of image pairs in training data set. This framework provides higher flexibility and can easily integrate a discriminative term to enhance the discriminability of the reconstructed image (to be discussed in Section 2.3).

\subsection{Discovering the Nonlinear Relationship}

Because of the nonlinear face variations, the relationship between LR images and HR images may be nonlinear and complicated. There does not exist closeform solution for finding $\mathcal{R}$, so that estimating $\mathcal{R}$ by Eq.(3) is not feasible. In this paper, we employ the nonlinear mapping $\Phi$ to map the original image to the high dimension feature space, as shown in Figure 1. In this feature space, the relationship between HR and LR features can be better modeled by linear approximation. That means in this feature space, the relationship operator $\mathcal{R}$ can be approximated by a matrix $R$. Following Eq.(3), $R$ can be estimated by

$$
R=\arg \min _{R^{\prime}} \sum_{i=1}^{N}\left\|R^{\prime} \Phi_{L}\left(I_{l}^{i}\right)-\Phi_{H}\left(I_{h}^{i}\right)\right\|^{2}
$$

Let $\mathbf{E}_{H}=\left\{e_{H}^{1}, e_{H}^{2}, \cdots\right\}$ and $\mathbf{E}_{L}=\left\{e_{L}^{1}, e_{L}^{2}, \cdots\right\}$ are the orthonormal bases of HR image feature space and LR image feature space. So we have:

$$
\begin{aligned}
& \min \sum_{i=1}^{N}\left\|R \Phi_{L}\left(I_{l}^{i}\right)-\Phi_{H}\left(I_{h}^{i}\right)\right\|^{2} \\
= & \min \sum_{i=1}^{N} \| R \sum_{j}<\Phi_{L}\left(I_{l}^{i}\right), e_{L}^{j}>e_{L}^{j} \\
& -\sum_{k}<\Phi_{H}\left(I_{h}^{i}\right), e_{H}^{k}>e_{H}^{k} \|^{2} \\
= & \min \sum_{i=1}^{N}\left\|R \mathbf{E}_{L} f_{L}^{i}-\mathbf{E}_{H} f_{H}^{i}\right\|^{2} \\
= & \min \sum_{i=1}^{N}\left\|\mathbf{E}_{H}^{-1} R \mathbf{E}_{L} f_{L}^{i}-f_{H}^{i}\right\|^{2}
\end{aligned}
$$

where $f_{L}^{j}$ is the weight (coefficient) of image $I_{L}^{j}$ in feature space. Let $R^{*}=\mathbf{E}_{H}^{-1} R \mathbf{E}_{L}$, to determine $R$ is equivalent to determine $R^{*}$. And we have

$$
R^{*}=\arg \min _{R^{\prime}} \sum_{i=1}^{N}\left\|R^{\prime} f_{L}^{i}-f_{H}^{i}\right\|^{2}
$$

However, it is very computationally expensive to calculate the nonlinear mapping $\Phi$ explicitly due to the high dimensionality of the feature space, so it is not feasible to calculating the relationship $R^{*}$ by Eq.(6) directly. Kernel trick is used and kernel subspace is used to represent the features in the kernel feature space. Let $\mathbf{S}_{H}=\left\{S_{H}^{1}, S_{H}^{2}, \ldots, S_{H}^{k_{1}}\right\}$ and $\mathbf{S}_{L}=\left\{S_{L}^{1}, S_{L}^{2}, \ldots, S_{L}^{k_{2}}\right\}$ represent the the kernel subspaces for HR image and LR image space respectively. Replace $\mathbf{E}_{L}$ and $\mathbf{E}_{H}$ with $\mathbf{S}_{L}$ and $\mathbf{S}_{H}$, we have

$$
R=\arg \min _{R^{\prime}} \sum_{i=1}^{N}\left\|S_{H}^{\prime} R^{\prime} S_{L} \hat{f_{L}^{i}}-\hat{f_{H}^{i}}\right\|^{2}
$$

where $\hat{f}_{L}^{i}$ and $\hat{f}_{H}^{i}$ are the LR and HR kernel subspace coefficients for representing the image features, respectively. Under this subspace representation form, we 


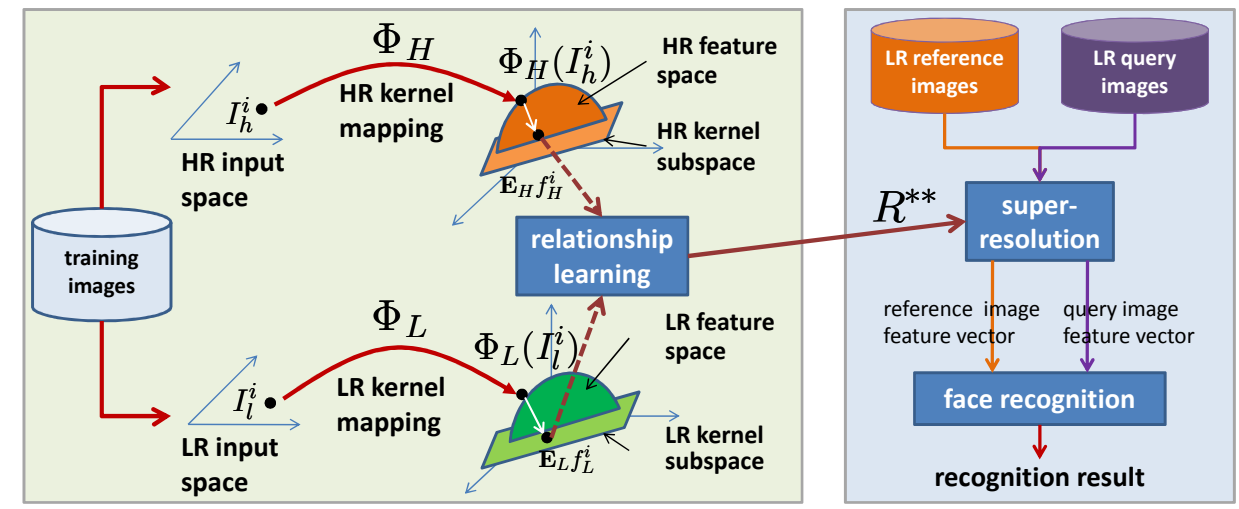

(a)

(b)

\section{Figure 1. Block diagram of proposed method: (a) the relationship training phase, (b) face recog- nition phase.}

learn the relationship between coefficients instead of features, so Eq.(6) becomes

$$
R^{*}=\arg \min _{R^{\prime}} \sum_{i=1}^{N}\left\|R^{\prime} \hat{f_{L}^{i}}-\hat{f_{H}^{i}}\right\|^{2}
$$

Given the LR query image, the HR image kernel subspace features can be reconstructed by

$$
\hat{f_{H}}=R^{*} \hat{f_{L}}
$$

After reconstructing the HR image kernel features, we can directly make use of those features for recognition using existing kernel based face recognition engine. Also, if the HR image is required, pre-image learning [12] can be adopted.

\subsection{Discriminative Relationship Learning}

Discriminability of the reconstructed HR image is important for recognition purpose. To achieve that, a discriminability cost function is designed so that the reconstructed HR images (features) are suitable for recognition.

A good way to enhance the discriminability is to make use of the label information of the training data. We expect the reconstructed HR images should be clustered with the images from the same class, and far away from the images from other classes. Inspired by the success of Maximum Margin Criterion, a discriminability cost function is developed as follows,

$$
\begin{aligned}
d(R)= & \sum_{k=1}^{K} \sum_{\hat{f}_{L}^{i} \in \Omega_{k}}\left\|R \hat{f}_{L}^{i}-\overline{\hat{f_{H}^{k}}}\right\|^{2} \\
& +\sum_{k=1}^{K} n_{k}\left\|R \overline{\hat{f_{L}^{k}}}-\overline{\hat{f_{H}}}\right\|^{2}
\end{aligned}
$$

where $\overline{\hat{f}} \frac{\overline{\hat{k}}}{L}\left(\overline{f_{H}^{k}}\right)$ is the mean of the features of LR (HR) images from class $\Omega_{k}$, while $\overline{\hat{f_{H}}}$ is the mean of the features of all HR training images; $n_{k}$ is the number of training images in class $\Omega_{k}$, while $K$ is the number of classes.

So the discriminative relationship learning can be conducted by minimizing the following equation.

$$
R^{* *}=\arg \min _{R^{\prime}} \sum_{i=1}^{N}\left\|R^{\prime} \hat{f_{L}^{i}}-\hat{f_{H}^{i}}\right\|^{2}+\alpha d\left(R^{\prime}\right)
$$

where $\alpha$ is weight to balance the reconstruction error and the discriminability cost function.

\section{Experimental Results}

KPCA [6] and KDDA [8] face recognition algorithms, as well as CMUPIE and FRGC V2.0 face databases are used for experiments. The resolution for the HR images and LR images are 56x64 and 14x16 respectively. To obtain the super-resolved images / features, the proposed super resolution algorithm is applied to LR image. Hallucination Face [1] (HF) and kernelbased face hallucination (KF) method [2] are also employed. The recognition algorithms are also applied to 


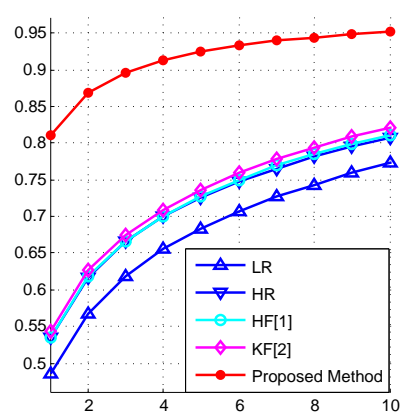

(a)

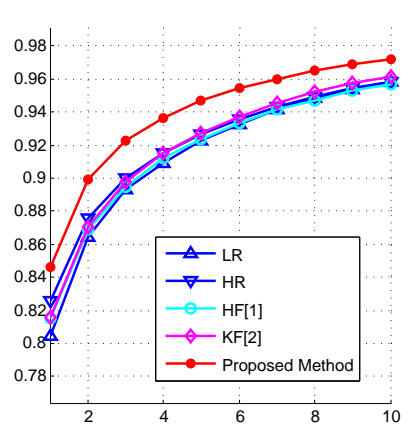

(b)

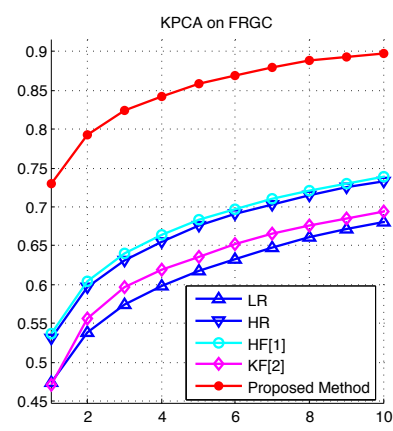

(c)

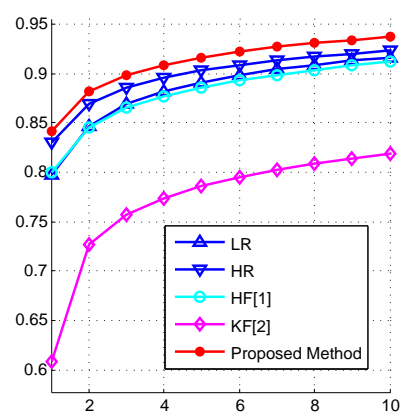

(d)

Figure 2. The CMC Curves of different face recognition methods on CMUPIE and FRGC database: (a) KPCA on CMUPIE (b) KDDA on CMUPIE (c) KPCA on FRGC (d) KDDA on FRGC

both HR and LR images and the results are used for comparison.

To estimate the effectiveness of the proposed method on dealing with nonlinear face variations, the face images used in our experiments contains face variations due to different poses, lighting conditions and expressions. In CMUPIE database, 21 lighting conditions and 5 poses are covered for each person (total 68 persons); in FRGC database, the training dataset is used, containing 12776 images (222 persons) with different pose, illumination and expression or a combination. Each database is divided into two non-overlapped sets. 10 images per person are randomly selected as training data which are used for learning the relationship $R^{* *}$ in Eq.(11) and the reference template while the rest of images are used as testing data.

The recognition results in terms of CMC curves are reported in Figure 2. It can be seen that, in general, the recognition based on HR images outperforms that of LR images. Recognition accuracy based on HR image generated by Hallucination Face (HF) method is close to that of HR images, which implies that HF method is good. Kernel-base face hallucination (KF) method, also performs better than LR in most cases. In all cases, recognition using the the HR image features generated by our proposed method gives the best results, which implies that the proposed method could handle both low resolution and non-linear variations well.

\section{Conclusion}

The problem of face hallucination with nonlinear face variations is discussed in this paper. To solve this problem, we have proposed a new face hallucination framework, which discovers the nonlinear relationship between the LR images and HR images with nonlinear face variations. The experimental results show that the proposed method significantly enhances the recognition performance.

Acknowledgement This project is partially supported by Science Faculty Research grant of Hong Kong Baptist University and NSFC-GuangdDong research grant U0835005.

\section{References}

[1] S. Baker and T. Kanade. Limits on super-resolution and how to break them. PAMI, 24(9):1167-1183, 2002.

[2] A. Chakrabarti, A. N. Rajagopalan, and R. Chellappa. Super-resolution of face images using kernel pca-based prior. IEEE Trans. on Multimedia, 9(4):888-892, 2007.

[3] B. Gunturk, A. Batur, Y. Altunbasak, M. Hayes, and R. Mersereau. Eigenface-domain super-resolution for face recognition. IEEE Trans. on Imag. Processing, 12(5):597-606, 2003.

[4] P. H. Hennings-Yeomans, S. Baker, and B. Kumar. Simultaneous super-resolution and feature extraction for recognition of low-resolution faces. In IEEE Intl. Conf. on CVPR, pages 1-8, 2008.

[5] K. Jia and S. Gong. Generalized face super-resolution. IEEE Trans. on Imag. Proc., 17(6):873-886, 2008.

[6] K. Kim, K. Jung, and H. Kim. Face recognition using kernel principal component analysis. IEEE Signal Processing Letters, 9(2):40-42, 2002.

[7] Y. Li and X. Lin. Face hallucination with pose variation. In 6th IEEE Intl. Conf. on AFGR, 2004. Proceedings, pages 723-728, 2004.

[8] J. Lu, K. Plataniotis, and A. Venetsanopoulos. Face recognition using kernel direct discriminant analysis algorithms. IEEE Trans. on Neural Networks, 14(1):117126, 2003.

[9] P. Mortazavian, J. Kittler, and W. Christmas. 3Dassisted Facial Texture Super-Resolution. In BMVC, 2009. 
[10] J. Yu, B. Bhanu, Y. Xu, and A. Roy-Chowdhury. Superresolved facial texture under changing pose and illumination. In Intl. Conf. on Image Processing, 2007.

[11] W. Zhao, R. Chellappa, P. Phillips, and A. Rosenfeld. Face recognition: A literature survey. ACM Computing Surveys (CSUR), 35(4):399-458, 2003.

[12] W.-S. Zheng, J. Lai, and P. C. Yuen. Penalized Preimage Learning in Kernel Principal Component Analysis. IEEE Trans. on Neural Networks, in Press.

[13] W. W. Zou and P. C. Yuen. Very Low Resolution Face Recognition Problem. Submitted to ECCV, 2010. 